\title{
Models for selecting an effective order fulfilment
}

\author{
Ivan Shidlovskii ${ }^{*}$ \\ ${ }^{1}$ HSE University, Department of Logistics Systems and Management, 119049 Shabolovka Ulitsa 26, \\ Moscow, Russia
}

\begin{abstract}
The problems of modelling cash flows are considered, which are determined by the procedures for optimising the order of execution of portfolio orders. The objective function assumes the maximisation of the average expected total income (accumulated on the deposit, taking into account the monetary amounts received from the implementation of portfolio orders) by a given point in time in the future. Considered approaches to the optimisation of such systems which allow taking into account: 1) the random nature of income from completed orders; 2) special additional costs, correlated both with the moment of the portfolio formation and with the moment of completion of order servicing.
\end{abstract}

\section{Introduction}

The paper considers methods, models and approaches to optimising solutions, which correlate with modifications of problems in the theory of service networks. Cash flows are modelled due to procedures for optimising the order of performing a given set of jobs (considered a portfolio of orders) to increase the efficiency of the analysed business processes. We are talking about tasks associated with the requirement to maximise the average expected total income (accumulated in the deposit account, taking into account the monetary amounts received from the implementation of portfolio orders) to a specific a priori given moment in the future. Other approaches to modelling cash flows in the format of problems of a similar type can be found, in particular, in $[3,4,8]$. At the same time, for particular tasks of choosing the order of performing the required operations, which correlate with the special topic of the problem of "multi-armed bandits", the corresponding methods are presented, for example, in $[5,6,7]$. Some of such approaches can be used for managing a fleet of shipping containers flowing [1]. A methodology based on analytical queueing networks coupled with nonlinear optimisation to design supply chain topologies and evaluate various performance measures is developed [9]. The problem of managing a homogeneous fleet of vehicles over time to serve a set of loads, each with a known origin and destination and a specified time window in which they must be served, is solved at [11].

The work will justify and present strategies for choosing the optimal order of queueing portfolio orders for special modifications of such optimisation models. They will allow practising managers to effectively use the specified hidden reserve of cost reduction, which correlate directly (or indirectly) with the analysed processes. In particular, the research

* Corresponding author: shdlvsk-ivan@yandex.ru 
presents developments that will make it possible to consider various special factors when optimising these solutions. These include:

- the need to take into account the random additional costs that occur at the time of the formation of a given portfolio of orders (for example, the cost of supplying materials, changeover of equipment, etc.), as well as immediately at the time of completion of order queueing procedures (loss of income, for example, due to the quality of work);

- the need to consider the relevant risks of accidental loss of income (concerning the format of the a priori agreed corresponding contractual amounts).

Of course, the ability to consider the specifics of the simulated cash flows for problems of this type. In addition, the possible additional costs/losses will undoubtedly provide managers with an essential research tool concerning optimisation procedures for systems of this type. Their optimisation can significantly affect the efficiency of the modelled supply chains.

Let us consider a generalisation of a special model of the optimisation problem associated with choosing the best order of queueing portfolio orders (see, for example, $[10,12])$. We will talk about a situation when the objective function involves maximising the average expected total income accumulated on the deposit (in the form of the corresponding sums of money received from the implementation of portfolio orders) by a given time in the future. The generalisation presented will allow practitioners to consider both the random nature of such received amounts and the possible special additional costs/losses noted above.

\section{Results and discussion}

Suppose we are talking about an already formed portfolio of $\mathrm{N}$ available orders. As for the models of the theory of queueing networks, we assume that only one order is executed at any time. The format of the model assumes that upon completion of queueing an $i$-order (order with number $i$ ), a certain amount of money will be paid. It is assumed that such an amount, on the one hand, is stipulated by the contract. However, the generalised model considered here, on the other hand, allows one to take into account an essential feature for practice: the size of such payments can be considered as random values.

Of course, the order of execution of portfolio orders will determine the structure of the incoming cash flow. It is assumed that we are talking about a model when the firm "works to accumulate the corresponding financial amounts of income/profit." The latter means that the indicated incomes are accumulated precisely on the deposit account of the firm by some time $T$ in the future. A priori is assumed that such a moment in time is undoubtedly (with probability one) greater than the time it takes for all orders in the portfolio to be completed. By the way, in this case, of course, it is also implicitly assumed that the company has the required funds that will be required to fulfil these orders. The deposit interest rate $r$ is known. We believe that the specified rate is set in annual terms. In this case, interest is accrued based on simple interest.

For all portfolio orders, optimisation should also consider the accompanying additional costs/losses of two types, correlated with different points in time (clarification is given below). In this case, here, we will consider the following generalised model for the problem, which is associated with the choice of the optimal order of queueing orders of a given portfolio.

It is required to determine such an order of execution of portfolio orders, in which the average expected amount of money on the deposit at a given time $T$ (which is the sum of all payments on orders) and taking into account both additional losses and the related accrued interest, will be maximum. Let us present the initially specified parameters of the considered model: 
- $S_{i}$ - the execution time of the $i$-order (these are independent random variables, with arbitrary laws of the probability distribution);

- $M\left[S_{i}\right]$ - an average lead time of the $i$-order;

- $\mu_{i}=1 / M\left[S_{i}\right]-$ the intensity of the $i$-order;

- $\vec{i}=\left(i_{1}, i_{2}, \ldots, i_{N}\right)-$ portfolio order execution vector;

- $\tilde{P}_{i}$ - random cash income that will be paid on the $i$-order at the time of completion of its execution (USD);

- $P_{i}$ - mathematical expectation for the corresponding paid amounts $\tilde{P}_{i}$ on income ( $i=$ $\overline{1, \mathrm{~N}})$ from the realised $i$-orders of the portfolio;

- $T$ - point in time to which financial savings are realised (years);

- $\tilde{T}_{i}$ - the moment of the end of the execution of the $i$-order, i.e. time of receipt of the corresponding amount on the deposit account $\tilde{P}_{i}$.

The format of the modified model under consideration, as already noted, will also allow taking into account special additional losses for $i$-orders (and not only random payments in the form of amounts $\tilde{P}_{i}$ ). To denote them, we will use the symbols $\tilde{\alpha}_{i}(1)$ and $\tilde{\alpha}_{i}(2)$, where

$\tilde{\alpha}_{i}(1)$ - the indicated losses, which will need to be correlated precisely with the moment of formation of the order portfolio, and in the general case, of course, they can also be random values (depending only on the type of order);

$\tilde{\alpha}_{i}(2)$ - the indicated losses will need to be correlated precisely with the time points of receipt of the corresponding monetary amounts of income $\tilde{P}_{i}$ on portfolio orders (also considered as random variables).

Let us formalise the components of the final total income by the time $T$ in the situation under consideration. It will be needed to take into account the following values (taking into account simple interest).

- For cash payments $\tilde{P}_{i}$ (from completed orders) by time $T$ the amount will be increased $\sum_{i=1}^{N} \tilde{P}_{i}\left(1+r\left(T-\tilde{T}_{i}\right)\right) \cdot$

- For total losses of type $\tilde{\alpha}_{i}(1)$ correlated with the moment of portfolio formation, it is necessary to consider the accumulated amount $\sum_{i=1}^{N} \tilde{\alpha}_{i}(1)[1+r T]$.

- Moreover, for total losses due to costs correlated with the points in time of payments of the amounts $\tilde{P}_{i}$ for $i$-orders of the portfolio, it is necessary to consider the accrued amount $\sum_{i=1}^{N} \tilde{\alpha}_{i}(2)\left[1+r\left(T-\tilde{T}_{i}\right)\right] \cdot$

Finally, for the average expected accumulated money amount on the deposit account by the time $T$ (we will denote it briefly as $D$ ), we can now note the following. The expression for the objective function under consideration can be represented as (this notation is formalised so that the first expression does not depend on the order of execution of portfolio orders):

$$
\mathrm{D}=M\left[\sum_{i=1}^{N}\left[\tilde{P}_{i}-\tilde{\alpha}_{i}(1)-\tilde{\alpha}_{i}(2)\right](1+r T)\right]-M\left[\sum_{i=1}^{N}\left[\tilde{P}_{i}-\tilde{\alpha}_{i}(2)\right] r \tilde{T}_{i}\right] \rightarrow \max ,
$$

moreover, maximisation should be achieved precisely and only by choosing the appropriate order of queueing portfolio orders. Standard simplification procedures lead to the following simpler (and more convenient for analysis) representation of the specified problem: 


$$
M\left[\sum_{i=1}^{N}\left[\tilde{P}_{i}-\tilde{\alpha}_{i}(2)\right] \tilde{T}_{i}\right] \rightarrow \min .
$$

The format of the optimisation problem of the order of selection of portfolio orders for queueing corresponds to the format of the related problem considered in [2]. This feature, of course, makes it possible to present the required attributes of the optimal strategy immediately.

When determining the best order of selecting portfolio orders for queueing and considering the specifics of the situation considered here, it is necessary to use the optimal $P \mu$-rule [2]. In this case, the required numerical indicators for the contract amounts (in the form of indicators $P_{i}$ in the format of the specified optimal $P \mu$-rule), naturally, will be necessary to replace, just, with the corresponding modified new indicators of the form: $M\left(\tilde{P}_{i}\right.$ $\left.-\tilde{\alpha}_{i}(2)\right)$.

\section{Conclusion}

The development of special procedures for optimising the order of execution of portfolio orders presented here will give in practice an opportunity to optimise such decisions, taking into account the following factors.

1. The ability to consider the risks correlated with the realisation of income on portfolio orders (in the considered model a priori, a random character is assumed for the paid contract amounts ).

2. The ability to consider special additional losses/costs, which are determined by the procedures for the implementation of portfolio orders, and correlate with the moment of formation of the portfolio (it was shown that they do not affect the optimal strategy for queueing orders).

3. The ability to take into account special additional losses/costs that are determined by the procedures for the implementation of portfolio orders, and, in particular, correlate with the moments of completion of the execution of orders (it has been shown that they significantly affect the optimal strategy since they affect the indicators important for optimisation of the kind $M\left(\widetilde{P}_{i}-\tilde{\alpha}_{i}(2)\right)$.

4. The optimal strategy for queueing orders can be determined based on the optimal $P \mu$ rule. The values of the parameters $P_{i}$ should be determined as the above indicators $M\left(\tilde{P}_{i}\right.$ $\left.\left.\tilde{\alpha}_{i}(2)\right)\right)$.

5. The proposed approach to generalisation/modification of the optimal $P \mu$-rule (taking into account the above factors) expands the range of problems that can be solved in modelling and optimising cash flows, correlated with implementing order portfolios in order to increase the efficiency of the corresponding systems.

\section{References}

1. D. Adelman, Price-Directed Control of a Closed Logistics Queueing Network, Operations Research, v. 55(6), pp. 1022-1038 (2007)

2. G.L. Brodetskiy, Optimal Service Order in Supply Chains, Taking Into Account the Risks of Loss and Inflation, Logistics and Supply Chain Management, v. 5, pp. 40-51 (2009)

3. F. Caro, J. Gallen, Dynamic Assortment with Demand Learning for Seasonal Consumer Goods. UCLA Anderson School of Management, Los Angeles, CA (2006) 
4. S. Chick, N. Gans, An Economic Analysis of Simulation Selection Problems. OPIM Department Wharton University of Pennsylvania (2006)

5. N. Gans, G. Knox and R. Groson, Simple Models of Discrete Choice and Their Performance in Bandit Experiments. The Wharton School, University of Pennsylvania (2004)

6. J.C. Gittins, Bandit Processes and Dynamic Allocation Indices, J. R. Statist. Sos. v. 41(2) (1979)

7. J.C. Gittins, Multi-Armed Bandit Allocation Indices. New York: Wiley, (1989)

8. S. Kavadias, C. Loch, Optimal Project Sequencing with Recourse at a Scarce Resource, Production and Operations Management, v. 12(4) (2003)

9. L. Kerbache and S.J. MacGregor, Queueing networks and the topological design of supply chain systems, International Journal of Production Economics, v. 91(3), pp. 251-272 (2004)

10. J. Walrand, An introduction to queueing networks. Englewood Cliffs, N.J., Prentice Hall (1988)

11. P.B. Warren and C.A. Tassio, Dynamic Control of Logistics Queueing Networks for Large-Scale Fleet Management, Transportation Science, v. 32(2), pp. 90-109 (1998)

12. R.J. Boucherie and N.M. van Dijk, Queueing Networks: A Fundamental Approach, International Series in Operations Research \& Management Science, Springer, New York (2011) 